\title{
Two Newly Recorded Spiders from Japan, Bavia sexpunctata (Doleschall, 1859) and Rhene setipes ŻABKA, 1985 (Araneae: Salticidae)
}

\author{
Akio TANIKAWA ${ }^{1)}$ \\ 谷川明男 ${ }^{1)}$ ：日本新記録種 Bavia sexpunctata (DOLESCHALL, 1859) \\ および Rhene setipes ŻABKA, 1985 そついて
}

\begin{abstract}
Two salticid spiders, Bavia sexpunctata (Doleschall, 1859) and Rhene setipes ŻABKA, 1985, are newly recorded from Japan. The genus Bavia is new to the Japanese fauna. The female of Rhene setipes is described for the first time.
\end{abstract}

After an examination of the materials obtained from Iriomotejima Island, Okinawa Pref., Japan, the author recognized the occurrence of Bavia sexpunctata (DolesChall, 1859) and Rhene setipes ŻABKA, 1985, in the island. These species are new to the Japanese fauna, and will be redescribed in the present paper.

Bavia sexpunctata was originally described by DOLESCHALL (1859) from Amboina. Then it has been revealed by many authors to be widely distributed from Sumatra to New Guinea (ThORell, 1878, 1881, 1890, 1892; КосH, 1879; Hasselt, 1893; SimON, 1899; Strand, 1911; ReIMOSER, 1927). The present author collected some specimens of this spider in a meadow by the sweeping method. The genus Bavia has never been recorded from Japan.

Rhene setipes was described from Viet-Nam by ŻABKA (1985) on the basis of the male holotype. The author could collect two females and one male in a forest by the beating method. The female of the species will be described for the first time in the present paper.

Before going further, the author wishes to express his hearty thanks to Dr. Hirotsugu OnO, National Science Museum (Nat. Hist.), Tokyo, for his constant guidance and revising the manuscript of this paper.

\section{Bavia sexpunctata (DOLESCHALL, 1859)}

(Figs. 1-7)

Salticus sexpunctatus Doleschall, 1859, p. 24 [male holotype (?) from Amboina, deposited in

Rijksmuseum van Natuurlijke Historie, Leiden (?), not examined].

Marptusa sexpunctata: Thorell, 1878, p. 224; 1881, p. 444; 1892, p. 288._-Hasselt, 1893, p. 156.

1) Shichirigahama Senior High School, 2-3-1, Shichirigahama-higashi, Kamakura-shi, Kanagawa, 248 Japan

神奈川県立七里ガ浜高等学校 T248 神奈川県鎌倉市七里ガ浜東 2-3-1

Accepted May 1, 1993 
Acompse dulcinervis L. KocH, 1879, p. 1149, tab. 100, figs. 1-2 [syntypes from Pelew (Palau) Islands, deposited in Zoologisches Institut und Zoologisches Museum der Universität, Hamburg, not examined], synonymized by THORELL (1881).

Bavia sexpunctata: THORELl, 1890, p. 75.—-SimON, 1899, p. 103.—STRAND, 1911, p. 179.ReIMOSER, 1927, p. 2.—PRÓSZYŃSKI, 1984, p. 13 (partim, male).—Ż̇ABKA, 1988, p. 436, figs. $40-45$.

Specimens examined. 1우, Funaura, Iriomotejima Island, Okinawa Pref., Japan, 19-VIII-1985, A. TANIKAWA leg.; 1우, Funaura, Iriomotejima Island, Okinawa Pref., Japan, 1-I-1987, A. TANIKAWA leg.; 10`, Urauchi, Iriomotejima Island, Okinawa Pref., Japan, 28-XII-1989, A. TANIKAWA leg.

Description (based on the Japanese specimens). Measurement (in mm). Body length 우 8.60-9.30, 워 8.55; carapace length 우 3.88-4.30, ơ 3.64, width 우 3.083.40 , ㅇ 3.00; abdomen length 우 4.40-5.00, o 4.35 , width 우 1.93-2.28, 귀 1.98 . Length of legs of 1 우 $10^{7}$ from Iriomotejima Island as shown in Table 1.

Female. Carapace length/width 1.26. Length of eye field/length of carapace 0.53 , eye field length/width $0.87-0.88$, width of eye field III/width of eye field I
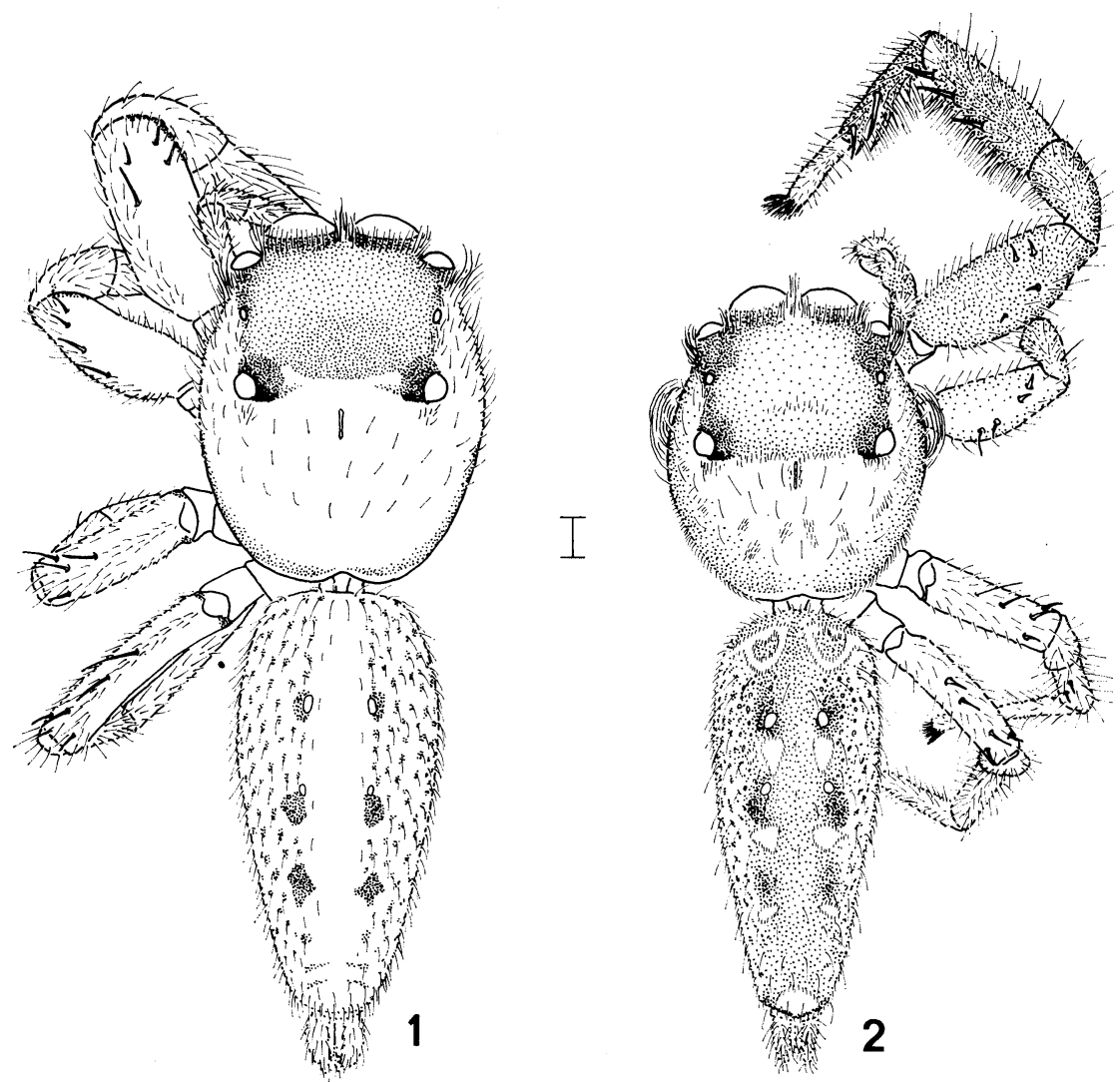

Figs. 1-2. Bavia sexpunctata (Doleschall, 1859)._-1. Female, dorsal view. 2. Male, dorsal view. (Scale: $0.5 \mathrm{~mm}$.) 
Table 1. Measurement of leg segments of Bavia sexpunctata

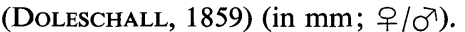

\begin{tabular}{lcccccc}
\hline Leg & Tarsus & Metatarsus & Tibia & Patella & Femur & Total \\
\hline I & $0.75 / 0.93$ & $1.28 / 1.69$ & $1.83 / 2.31$ & $1.54 / 1.63$ & $2.28 / 2.64$ & $7.68 / 9.20$ \\
II & $0.68 / 0.69$ & $1.10 / 1.17$ & $1.25 / 1.31$ & $1.21 / 1.14$ & $1.90 / 1.94$ & $6.14 / 6.25$ \\
III & $0.63 / 0.63$ & $1.30 / 1.31$ & $0.93 / 0.94$ & $1.03 / 0.99$ & $1.73 / 1.79$ & $5.62 / 5.66$ \\
IV & $0.68 / 0.66$ & $1.83 / 1.84$ & $1.69 / 1.70$ & $1.13 / 1.06$ & $2.26 / 2.24$ & $7.59 / 7.50$ \\
\hline
\end{tabular}

1.01-1.03, distance between eye field I and II/distance between eye field II and III $0.82-0.93$, width of eye field III/width of carapace 0.76 . Thoracic furrow longitudinal. Chelicera with 4 promarginal and 7 retromarginal teeth. Labium length/ width 1.22-1.41, sternum length/width 1.52-1.54. Length of leg $\mathrm{I} /$ length of cara-
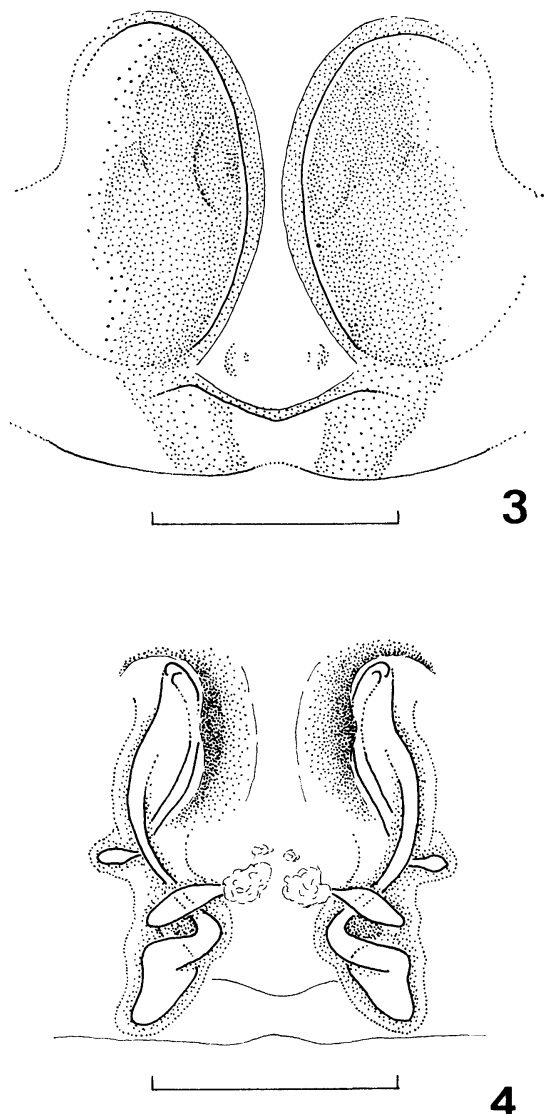

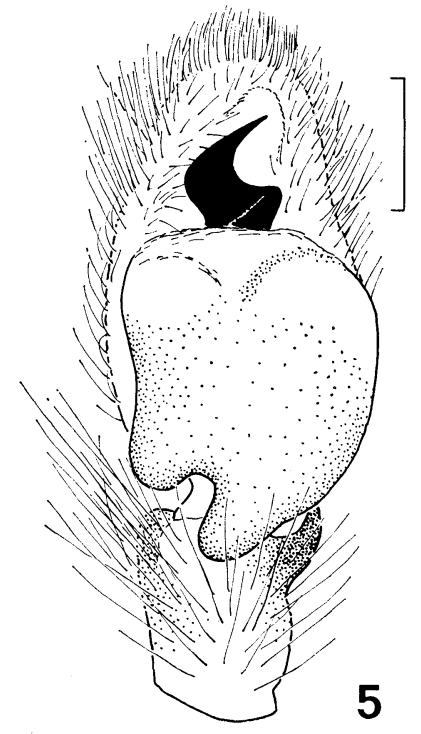

5

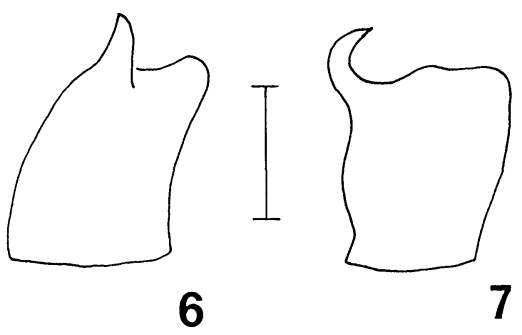

Figs. 3-7. Bavia sexpunctata (Doleschall, 1859)._-3. Epigynum. 4. Female genitalia, dorsal view. 5. Male palp, ventral view. 6. Tibia of male palp, retrolateral view. 7. Same, dorsal view. (Scales: $0.25 \mathrm{~mm}$.) 
pace 1.97-2.09. Abdomen length/width 2.19-2.28. Epigynum and internal genitalia as shown in Figs. 3-4.

Male. Carapace length/width 1.21. Length of eye field/length of carapace 0.57 , eye field length/width 0.87 , width of eye field III/width of eye field I 1.03 , distance between eye field I and II/distance between eye field II and III 0.86 , width of eye field III/width of carapace 0.79 . Thoracic furrow longitudinal. Chelicera with 4 promarginal and 7 retromarginal teeth. Labium length/width 1.32, sternum length/ width 1.52. Length of leg $\mathrm{I} /$ length of carapace 2.53. Male palp as shown in Figs. 5-7. Abdomen length/width 2.20.

Coloration and markings. Female. Carapace dark brown, surroundings of eyes black. Leg I dark brown, except light brown tarsus, legs II, III, IV light brown; palp light brown with dorsal dark brown line. Abdomen light brown, with numerous small dark brown spots and three pairs of larger ones.

Male. Carapace dark brown, surroundings of eyes black. Leg I dark brown, except light brown tarsus, legs II, III, IV brown. Abdomen light brown, mottled with dark brown, with three pairs of pale brown spots.

Range. Sumatra (ThORELl, 1892; Hasselt, 1893; Simon, 1899; ReImOSER, 1927), Buru (ThORell, 1881), Amboina (Doleschall, 1859; ThORell, 1878), Banda (Strand, 1911), Aru (Thorell, 1881), Palau (Koch, 1879), New Guinea (Thorell, 1881), Japan (new record; Iriomotejima Island).
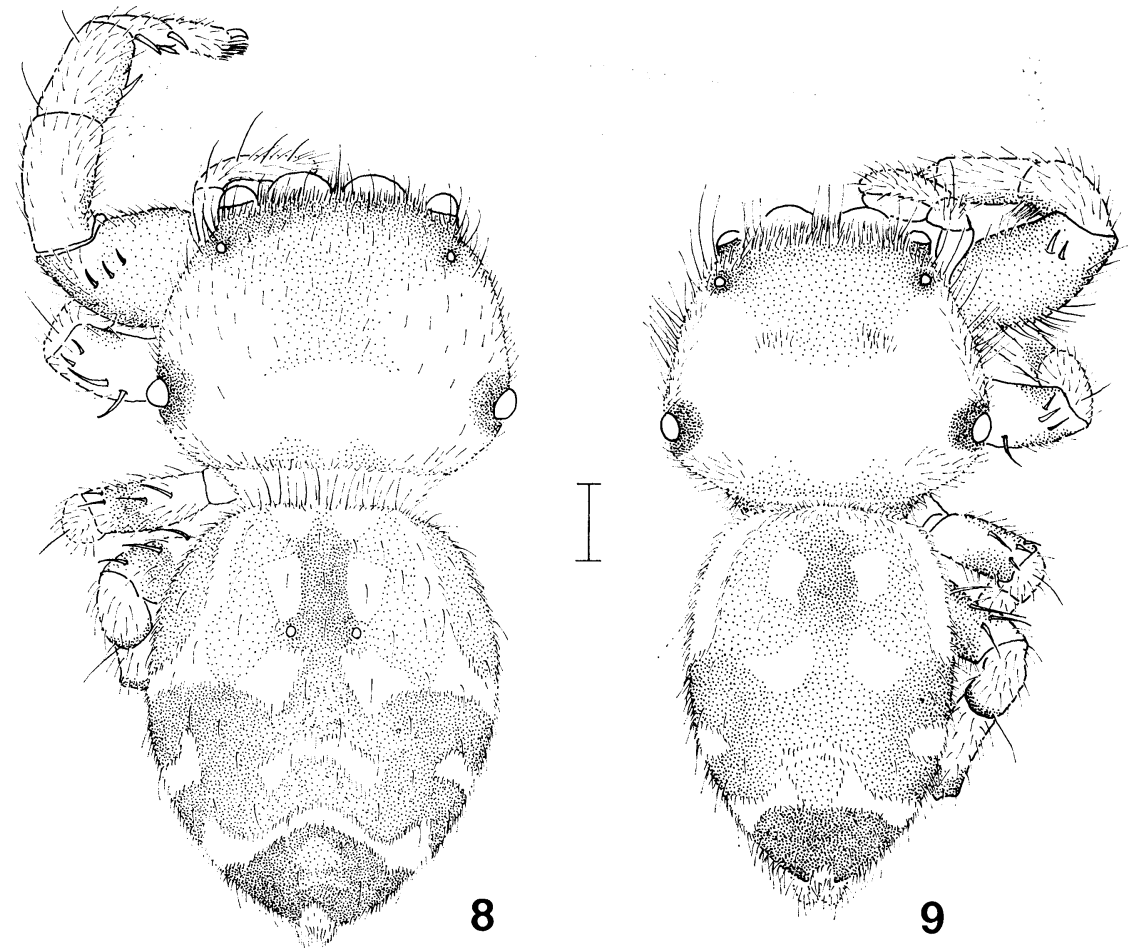

Figs. 8-9. Rhene setipes $\dot{Z}_{\mathrm{ABKA}}$, 1985.-8. Female, dorsal view. 9. Male, dorsal view. (Scale: $0.5 \mathrm{~mm}$.) 
Rhene setipes $\dot{Z}_{\mathrm{ABKA}}, 1985$

(Figs. 8-14)

Rhene setipes ŻABKA, 1985, p. 445, figs. 563-566 [male holotype from Luc Yen, Yen Bai Prov., VietNam, 5-XII-1971, TOPÁL \& MATSKÁsI leg., deposited in Thermészettudományi Múzeum, Budapest, not examined].

Specimens examined. 1오, Komi, Iriomotejima Island, Okinawa Pref., Japan, 16-VIII-1985, A. TANIKAWA leg.; 1우, Funaura, Iriomotejima Island, Okinawa Pref., Japan, 19-VIII-1985, A. TANIKAWA leg.; 10', Komi, Iriomotejima Island, Okinawa Pref., Japan, 27-III-1987, A. TANiKAWA leg.
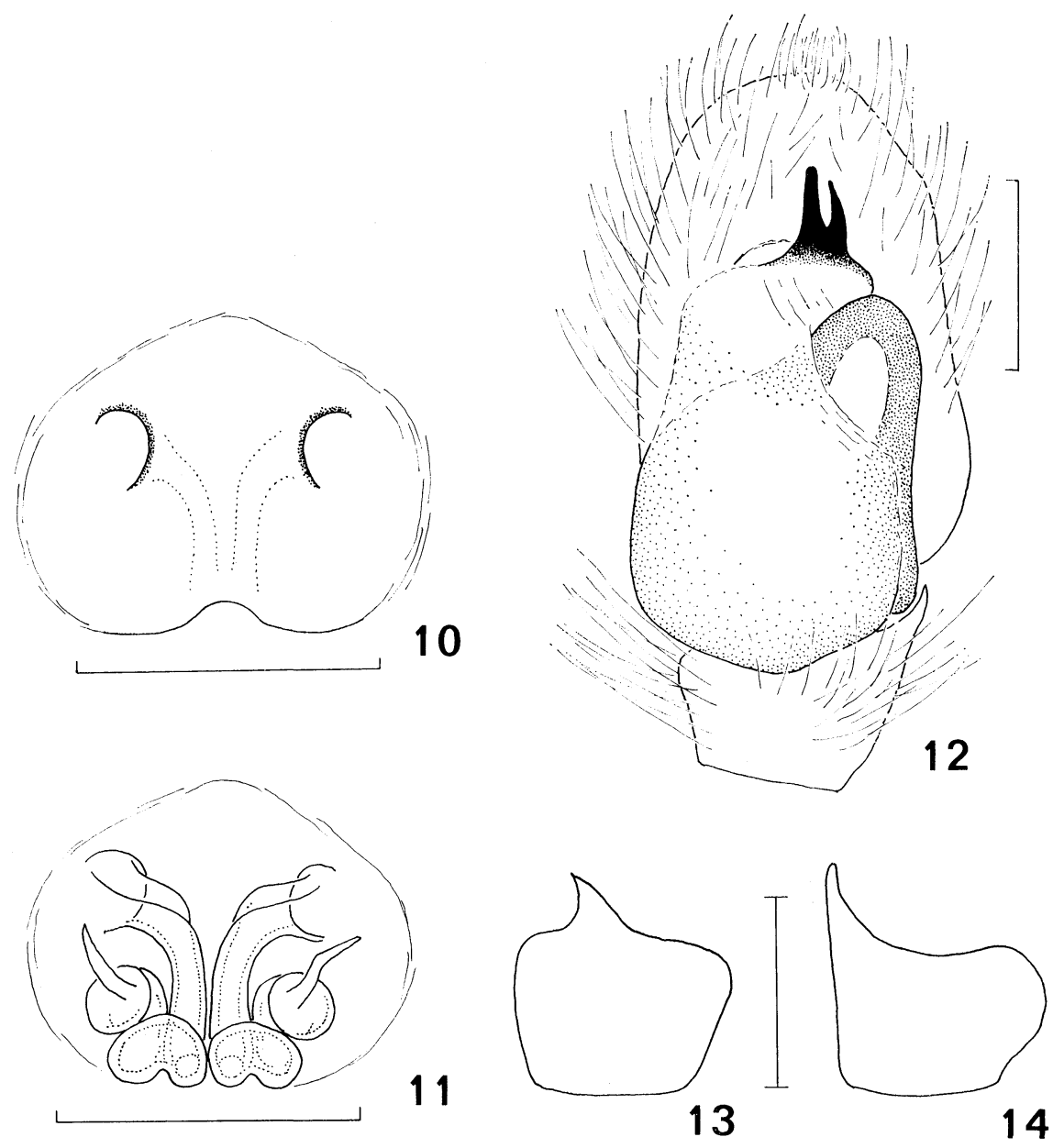

Figs. 10-14. Rhene setipes ŻंABKA, 1985.-10. Epigynum. 11. Female genitalia. 12. Male palp, dorsal view. 13. Tibia of male palp, retrolateral view. 14. Same, dorsal view. (Scales: $0.2 \mathrm{~mm}$.) 
Table 2. Measurement of leg segments of Rhene setipes $\dot{Z}_{\mathrm{ABKA}} 1985$ (in $\mathrm{mm}$; 우/ $/{ }^{7}$ ).

\begin{tabular}{lcccccc}
\hline Leg & Tarsus & Metatarsus & Tibia & Patella & Femur & Total \\
\hline I & $0.47 / 0.49$ & $0.48 / 0.57$ & $0.78 / 0.91$ & $0.87 / 0.92$ & $1.37 / 1.45$ & $3.88 / 4.34$ \\
II & $0.37 / 0.38$ & $0.48 / 0.52$ & $0.53 / 0.53$ & $0.63 / 0.61$ & $1.05 / 1.00$ & $3.06 / 3.04$ \\
III & $0.37 / 0.38$ & $0.53 / 0.53$ & $0.42 / 0.43$ & $0.52 / 0.50$ & $0.97 / 0.95$ & $2.81 / 2.79$ \\
IV & $0.38 / 0.38$ & $0.69 / 0.68$ & $0.68 / 0.63$ & $0.67 / 0.62$ & $1.27 / 1.17$ & $3.69 / 3.48$ \\
\hline
\end{tabular}

Description (based on the Japanese specimens). Measurement (in $\mathrm{mm}$ ). Body length 우 4.30-4.65, 위 4.25; carapace length 우 1.91-2.15, o 2.13 , width 우 1.892.20, 어 2.05; abdomen length 우 2.45-2.63, 위 2.43, width 우 1.80-2.37, 위 1.78 . Length of legs of 1 우 $10^{\top}$ from Iriomotejima Island as shown in Table 2.

Female. Carapace length/width $0.98-1.01$. Length of eye field/length of carapace $0.73-0.77$, eye field length/width $0.70-0.75$, width of eye field III/width of eye field I 1.48, distance between eye field I and II/distance between eye field II and III 0.26, width of eye field III/width of carapace 1.02-1.04. Thoracic furrow longitudinal. Chelicera with 2 promarginal and 1 retromarginal teeth. Labium length/ width 1.31-1.35, sternum length/width 1.59-1.71. Length of leg $\mathrm{I} /$ length of carapace 1.85-1.86. Abdomen length/width 1.11-1.36. Epigynum and internal genitalia as shown in Figs. 10-11.

Male. Carapace length/width 1.04. Length of eye field/length of carapace 0.70 , eye field length/width 0.70 , width of eye field III/width of eye field I 1.48 , distance between eye field I and II/distance between eye field II and III 0.23, width of eye field III/width of carapace 1.04. Thoracic furrow longitudinal. Chelicera with 2 promarginal and 1 retromarginal teeth. Labium length/width 1.25 , sternum length/ width 1.58. Length of leg I/length of carapace 2.04. Male palp as shown in Figs. 12 14. Abdomen length/width 1.37.

Coloration and markings. Female. Carapace dark brown, darker in front, surroundings of eyes black. Abdomen pale brown, with dark brown markings.

Male. Carapace dark brown, darker in front, surroundings of eyes black. Abdomen reddish brown, with dark brown and pale brown markings.

Range. Viet-Nam (ŻABKA, 1985), Japan (new record; Iriomotejima Island).

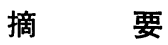

西表島より採集された Bavia sexpunctata（Doleschall, 1859）ムッボシハェトリ（新 称）および Rhene setipes ŻABKA, 1985 ミナミカラスハエトリ（新称）の2 種を日本新 記録として報告した。Bavia ムッボシハエトリグモ属（新称）は日本新記録属となる。ま た, Rhene setipes $\dot{Z}_{\mathrm{ABKA}}, 1985$ ミナミカラスハエトリの雌を初めて記載した.

\section{References}

Doleschall, C. L., 1859. Tweede Bijdrage tot de Kennis der Arachniden van den Indsche Archipel. Act. Soc. sci. Ind.-Neerl., 5: 1-60.

Hasselt, A. W. M. van, 1893. Spinnen van Java, Sumatra, en Ceylon voor den Heer J. R. H. 
Neervoortvan de Poll door den Heer J. Z. Kannegieter aldaar verzameld. Tijdschr. Ent., 36: $129-158$.

Косн, L., 1879. Die Arachniden Australiens, nach der Natur beschrieben und abgebildet, pp. 1045-1156. Bauer \& Rapse, Nürnberg.

Prószyński, J., 1984. Atlas rysunków diagnostycznych mniej znanych Salticidae (Araneae). Zesz. nauk. W.S.R.-P., 2: 1-177.

ReImoser, E., 1927. Spinnen von Sumatras Ostküste. Misc. zool. Sumatr., 13: 1-6.

Simon, E., 1899. Contribution à la faune de Sumatra. Arachnides recueillis par M. J. L. WeYers, à Sumatra. Ann. Soc. ent. Belg., 43: 78-125.

Strand, E., 1911. Araneae von den Aru- und Kei-Inseln. Abh. senckenb. naturf. Ges., 34: 129_ 199.

Thorell, T., 1878. Studi sui Ragni Malesi e Papuani. Part II. Ragni di Amboina raccolti dal Prof. O. Beccari. Ann. Mus. civ. Stor. nat. Genova, 13: 1-317.

- 1881. Ditto. Part III. Ragni dell'Austro-Malesia e del Capo York, conservati nel Museo civico di Storia naturale di Genova. Ibid., 17: VII-XXVII, 1-720.

1890. Arachnidi di Nias e di Sumatra raccolti nel 1886 dal Sig. E. Modigliani. Ibid., 30: 5-106 (not seen).

1892. Studi sui Ragni Malesi e Papuani. Part IV, 2. Ibid., 31: 1-490.

ŻABKA, M., 1985. Systematic and zoogeographic study on the family Salticidae (Araneae) from Viet-Nam. Annls. zool., 39: 197-485.

1988. Salticidae (Araneae) of Oriental, Australian and Pacific Regions, III. Ibid., 41: $421-479$. 